\title{
An evidence-based review of natalizumab therapy in the management of Crohn's disease
}

This article was published in the following Dove Press journal:

Therapeutics and Clinical Risk Management

20 November 2009

Number of times this article has been viewed

\author{
Raja GR Edula \\ Michael F Picco \\ Mayo Clinic, Jacksonville, Florida, USA
}

\begin{abstract}
Treatment options for Crohn's disease have evolved beyond the early goals of induction and remission and are now more focused on preventing complications by altering the natural history of the disease. The advent of biologic therapies has revolutionized the management of Crohn's disease. Specifically, antibodies to tumor necrosis factor alpha induce rapid mucosal healing. This translates into improved patient outcomes. However, many patients will fail these and other therapies. Natalizumab is a new biologic agent that has been approved for the treatment of moderately to severely active Crohn's disease in patients who have failed or are intolerant to immunosuppressants and/or tumor necrosis factor inhibitors. It is a selective adhesion molecule inhibitor to alpha-4 integrin resulting in inhibition of the migration of inflammatory cells across the endothelium. This unique mechanism of action has been shown to be effective in the treatment of Crohn's disease, making it an important option for otherwise refractory patients. Its use has been limited to these refractory patients because of concerns about the development of complications, especially progressive multifocal leukoencephalopathy. In this review, evidence-based data on the indications, efficacy and safety of natalizumab will be presented and its role in the management of patients with Crohn's disease will be defined.
\end{abstract}

Keywords: Crohn's disease, natalizumab, alpha-4 integrin

\section{Introduction}

Crohn's disease is a heterogeneous chronic inflammatory disease of the gastrointestinal tract defined based on endoscopic, histological and radiographic criteria. It can manifest as focal or patchy inflammation confined to the bowel wall or result in complications such as fistula leading to abscess, or stricture leading to chronic intermittent bowel obstruction. Like many other chronic diseases, Crohn's disease has no definitive cure and requires approaches to induce and maintain remission, improve quality of life and prevent complications that may occur in the long or short term, either related to medication use or directly as a result of the disease. ${ }^{1}$ Current cost of medical and surgical therapy is estimated at US\$2 billion annually. ${ }^{2,3}$

Selection of therapy is based on disease location, behavior and severity. ${ }^{4}$ Biological therapies especially inhibitors of tumor necrosis factor have revolutionized the management of Crohn's disease. Natalizumab is the first non-anti-TNF alpha biological agent approved for the treatment of Crohn's disease with a unique mechanism of action. It will be the focus of this review.
Correspondence: Michael F Picco

Division of Gastroenterology, Mayo

Clinic, 4500 San Pablo Rd., Jacksonville

FL, 32224, USA

$\mathrm{Tel}+\mathrm{I}(904) 953-2453$

Fax + I (904) 953-7260

Email picco.michael@mayo.edu 


\section{Disease classification and behavior}

Patients with Crohn's disease are classified based on the Montreal classification (Table 1) which includes of age at diagnosis, disease location and behavior. ${ }^{5}$ This classification has important implications for prognosis and therapy. Response rates to medical therapy are different based on disease location or behavior, with some medications effective only for disease confined to the lining of the bowel (non-stricturing, non-penetrating) whereas others are more effective for penetrating disease. ${ }^{1}$ Medical therapy is ineffective for stricturing disease which leads to chronic scarring that may result in bowel obstruction requiring surgery. Crohn's disease behavior may change with time with the progression of disease confined to the bowel wall to stricturing or penetrating (fistulizing) disease. Perianal manifestations are unique to Crohn's disease and may precede the onset of actual bowel symptoms. ${ }^{6}$ Gastric and duodenal manifestations are rare but increasingly being recognized. Extra-intestinal manifestations include spondylarthritis, peripheral arthritis, cutaneous manifestations (erythema nodosum, pyoderma gangrenosum), ocular manifestations (uveitis, episcleritis), primary sclerosing cholangitis and hypercoagulability. Long duration of disease can be complicated by gastrointestinal malignancy., ${ }^{7,8}$

Risk factors for predicting early development of severe Crohn's disease include presence of perianal disease, need for corticosteroids to treat first flare up and ileo- colonic location. ${ }^{9}$ Age of onset less than 40 is not a good predictor for early development of severe disease. Stricturing behavior and weight loss greater than $5 \mathrm{~kg}$ at diagnosis were independently associated with early development of severe disease. ${ }^{9}$

Table I The Montreal classification of Crohn's disease ${ }^{5}$

\begin{tabular}{ll}
\hline Age at diagnosis (A) & Al below 16 years \\
& A2 between 17 and 40 years \\
& A3 above 40 years \\
Location (L) & LI ileal \\
L2 colonic \\
L3 ileocolonic \\
L4 isolated upper disease \\
Behavior (B) & BI non-stricturing non-penetrating \\
& B2 stricturing \\
& B3 penetrating \\
P perianal disease modifier & \\
\hline
\end{tabular}

Notes: ${ }^{\mathrm{L}} \mathrm{L}$ is a modifier that can be added to $\mathrm{LI}-\mathrm{L} 3$ when concomitant upper disease is present. " $\mathrm{P}$ " is added to $\mathrm{BI}-\mathrm{B} 3$ when concomitant perianal disease is present.
However, predictive models remain imperfect due to the complex heterogeneity of this disease.

\section{Therapeutic approach to Crohn's disease}

Treatment options for Crohn's disease are individualized to disease location, behavior and previous medication use. The goals of therapy are to induce and maintain remission and also improve the natural history of the disease and avoid surgery. ${ }^{1}$ Corticosteroids while effective in inducing remission are not effective for remission maintenance and their benefit is only among patients with non-stricturing non-penetrating disease. They have significant short and long-term side effects and prolonged use should be avoided. Immune suppressants including azathioprine, 6-mercaptopurine and methotrexate typically take weeks or months to become effective and are not used for induction of remission but for remission maintenance. ${ }^{4}$ Inhibitors of tumor necrosis factor (TNF) alpha (infliximab, adalimumab and certolizumab) are biologic agents that are effective for rapid induction of remission and maintenance of remission among patients with non-stricturing non-penetrating disease or penetrating disease. Mesalamine agents, while useful in ulcerative colitis, are generally ineffective in Crohn's disease except in some cases of Crohn's colitis. Antibiotics, especially metronidazole, are effective in decreasing drainage of fistula and are helpful in the management of Crohn's disease but are not usually given as monotherapy. ${ }^{10}$ Surgery is indicated for disease not responding to medical treatment, obstruction secondary to stricturing disease, suppurative complications and histologic dysplasia or malignancy.

Current therapy for Crohn's disease has moved beyond the goal of induction and remission and now focuses on preventing complications. This "top down" approach which involves early introduction of immune modulators and biological agents is gaining popularity. ${ }^{11}$ Inhibitors of TNF induce rapid and profound endoscopic healing of mucosal lesions, which translates to improved quality of life, avoidance of hospitalization and surgery and prevention long term complications. ${ }^{12}$

Unfortunately, as with other therapies a significant number of patients are unresponsive, lose response or develop intolerance to these medications. For patients who initially respond, on average $37 \%$ will lose response or a rate of $13 \%$ per patient-year of treatment. ${ }^{13}$ These calculations were based on studies with up to a year of follow-up. Recent data from Belgium have been more encouraging, with nearly twothirds of patients maintaining benefit after nearly 5 years of follow-up. ${ }^{14}$ Improved sustained response was likely due 
to dose escalation of infliximab or shortening of the dosing interval. However one-third of patients still lost response. Many patients who respond to infliximab will need a higher dose, a more frequent dosing schedule or both, to maintain response. Most of these patients will demonstrate clinical response but a third of these will require alternative therapy. This proportion may increase as patients are treated for longer periods with these agents. Patients who have lost response to one agent may respond to a second TNF antagonist. Among patients who had failed infliximab, 52\% responded to adalimumab but remission rates were only $20 \% .{ }^{15}$ These patients who fail anti-TNF therapies may potentially benefit from natalizumab therapy.

\section{Natalizumab mechanism of action}

Alpha-4 integrin is a selective adhesion molecule that facilitates adhesion and subsequent leukocyte migration into areas of inflammation. Natalizumab (Tysabri ${ }^{\circledR}$; Elan) is a recombinant, humanized, IgG4 monoclonal antibody to alpha-4 integrin. ${ }^{16}$ Inhibition of integrins has been proven from animal studies to help in mucosal healing and reduce inflammation.

Leukocyte adhesion in endothelial cells is a multistep process that involves chemokine receptors and active integrins. Firm adhesion is the last step before leukocyte diapedesis through endothelial pores into the lamina propria of inflamed tissue. Natalizumab blocks both alpha-4 B1 integrin (VCAM 1) and alpha-4 B7 integrin (MADCAM 1) interactions. ${ }^{17}$ Its inhibition of leukocyte trafficking is not organ specific. In addition to the gut, other organs such as brain, bone marrow and kidney are also affected. This had lead to studies in diverse chronic inflammatory conditions such as multiple sclerosis and Crohn's disease.

\section{Natalizumab efficacy in multiple sclerosis}

Natalizumab is highly effective at preventing recurrent inflammation in patients with multiple sclerosis. The most striking effect is the reduction of contrast enhancing lesions on MRI and thus preventing clinical relapse. It is used in combination with interferon for treatment of relapsing and remitting multiple sclerosis. ${ }^{18}$

\section{Clinical studies of natalizumab in Crohn's disease}

Natalizumab is an effective therapy for treatment of adults with moderately to severely active Crohn's disease as defined by the Crohn's Disease Activity Index (CDAI). ${ }^{19}$ This conclusion is based on three randomized controlled trials. $^{20,21}$ Two of these large randomized controlled trials were published together. The first was an induction trail entitled Efficacy of Natalizumab as Active Crohn's Therapy (ENACT-1) and the second a maintenance trial entitled Evaluation of Natalizumab as Continuous Therapy (ENACT-2). In the first study patients were randomized to $300 \mathrm{mg}$ intravenous infusion of natalizumab or placebo at weeks 0,4 and 8 . The primary end point of this trial was clinical response as defined as a drop in the CDAI at week 10 of 70 points. Patients who responded were then eligible for the second study that randomized patients to $300 \mathrm{mg}$ intravenous natalizumab or placebo every 4 weeks through week 56 . The primary outcome of this study was sustained response at week 36. ENACT-1 showed a slight statistical improvement in response with natalizumab compared to placebo at week $10(56 \%$ vs $49 \%, P=0.05)$ but this was not clinically meaningful and there was no difference in remission rates $(37 \%$ vs $30 \%, P=0.12)$. Continuing natalizumab as maintenance therapy among responders led to high response rates and remission rates compared to placebo (61 vs 28\%: $P<0.001$ and 44 vs $26 \% ; P=0.003$, respectively) at week 36 . The minimal differences seen in ENACT-1 may have been due to patient selection since when the analysis was restricted to those with elevated C-reactive protein (indicating active disease) response and remission rates were higher. This led to a second study entitled Efficacy of Natalizumab in Crohn's Disease Response and Remission (ENCORE) that randomized only patients with elevated C- reactive protein to natalizumab or placebo at weeks 0,4 and 8 . Forty-eight percent of natalizumab treated patients had response at week 8 sustained to week 12 compared to $32 \%$ for placebo $(P<0.001)$ with remission rates $26 \%$ and $16 \%$ respectively $(P=0.002)$. Overall response and remission rates were higher in the natalizumab groups compared to placebo at 0,4 and 8 weeks. Corticosteroid tapering was more successful in the natalizumab group compared to the placebo group (58\% vs $28 \%$ ).

These studies support the efficacy of natalizumab for patients with moderately to severely active Crohn's disease both for induction and maintenance of remission and are summarized in Figure 1. While response rates compared to other agents for Crohn's disease were low, the patients enrolled in these studies differed in their disease severity and past medication usage. Over one-third of the patients in ENACT studies and half in the ENCORE studies previously had been exposed to antagonists to TNF and one-third in 


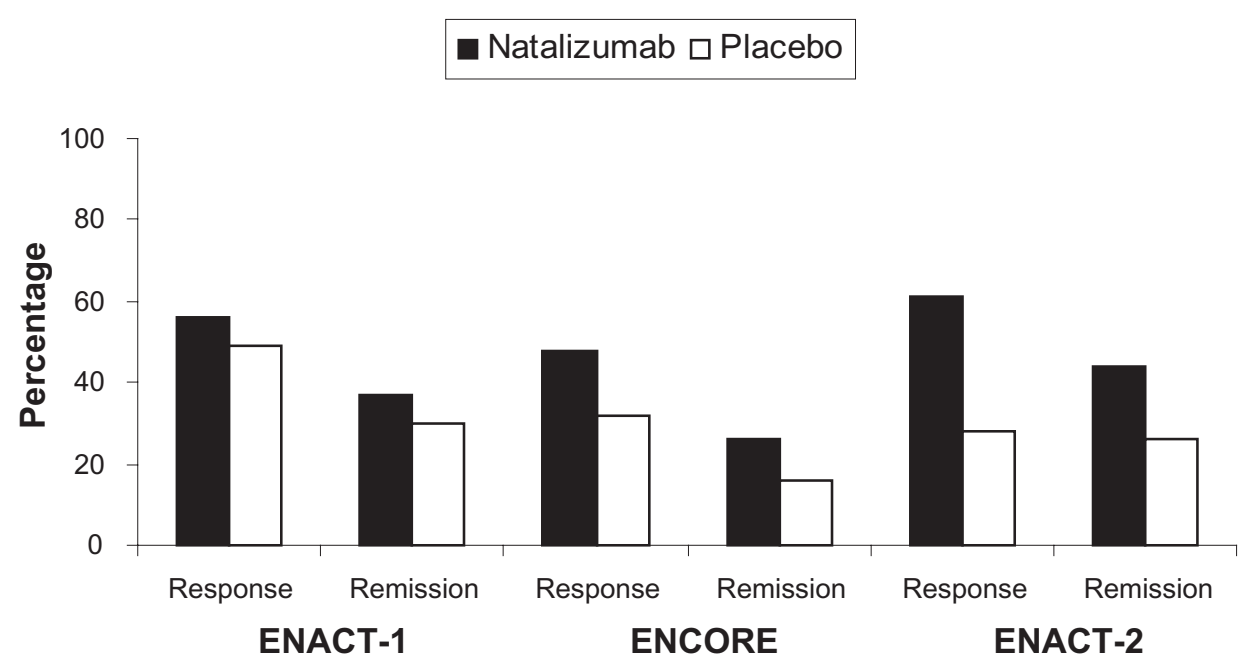

Figure I Comparison of response and remission rates for three pivotal studies for natalizumab in moderate to severely active Crohn's disease $(P=0.05$ and $P=0.12$ in ENACT-I, $P<0.001$ and $P=0.002$ in ENCORE and $P<0.001$ and $P=0.003$ in ENACT-2 for response and remission respectively).

both the studies received immunomodulator therapy during the trials. This suggests that these patients had more severe treatment refractory Crohn's disease.

Both ENACT and ENCORE studies had other important limitations. These studies were primarily focused on nonstricturing, non-penetrating disease and there is a lack of evidence for natalizumab in penetrating disease which is the primary limitation in application to the Crohn's disease population. While TNF inhibitors have been proven to be effective in fistula healing and closure, there are no data on natalizumab.

In addition, the patient population studied was limited to those who had severe refractory disease and most of them had already been on treatment with immune modulators or tumor necrosis factor inhibitors. Like other trials, they excluded patients with an ostomy, short bowel or stricture. The studies also lacked power to detect serious adverse events. It is also important to understand that only adult patients were included in these studies. One trial to date focused on adolescents with Crohn's disease and showed acceptable safety. Response rates were high but there was no placebo group. ${ }^{23}$ No study to date has shown any benefit of dosing based on patients weight or age. While C-reactive protein has not been shown to correlate well with disease severity in previous studies, ${ }^{24}$ in these natalizumab trials elevated levels did define a group of patients who were more likely to have a favorable response.

In the ENACT and ENCORE studies patients must not have received an antagonist to TNF for 3 months prior to study enrollment. In these trials patients who had previously received these agents were treatment failures. The question whether natalizumab can be administered with a TNF antagonist is potentially important. Clinicians may prefer to overlap therapy when a TNF antagonist is failing, to avoid severe disease exacerbations. The most important concern of this approach is safety. Short term safety and tolerability were confirmed among a population of patients receiving infliximab but not in remission who received natalizumab. ${ }^{25}$ However the study was small and improved therapeutic outcome could not be demonstrated and followup was less than 1 year. This study was initiated before any cases of progressive multifocal leukoencephalopathy (PML) were reported in Crohn's disease. This approach of overlapping TNF antagonist therapy with natalizumab can not be endorsed because of the short duration of this trial, its inability to detect rare important adverse events such as PML and concerns regarding significant immune suppression.

\section{Positioning of natalizumab}

Natalizumab has been approved for the treatment of mild to moderate Crohn's disease with evidence of inflammation in the United States. Since its mode of action is completely different from other biological agents, it is an important option for the treatment of Crohn's disease, among those patients who are intolerant or have lost efficacy to all other treatments including immune modulators and tumor necrosis factor inhibitors. Its restriction to use in these refractory cases is based on the risk of developing adverse events, particularly PML, which will be discussed further and current recommendation is that natalizumab should be administered as monotherapy (without concomitant immunosuppressive therapy) due to the risk of developing PML. 


\section{Safety and adverse effects}

The combined safety data for natalizumab in clinical studies suggest that the drug was generally well tolerated. Adverse events occurred infrequently and were experienced by a similar proportion of natalizumab and placebo-treated patients. There were no statistically significant differences in the proportions of patients who withdrew due to adverse events or those who experienced serious adverse events.

The most frequently reported adverse events with natalizumab infusion include headache, nausea, worsening of Crohn's disease and nasopharyngitis. Other events include abdominal pain, arthralgias, fatigue, hypersensitivity like reactions and the development of antibodies against natalizumab. When persistent anti-natalizumab antibodies were present, they appear to be associated with infusion reactions and loss of efficacy. Antibodies to natalizumab were detected in $8 \%$ and $9 \%$ of natalizumab exposed patients in ENACT-1 and ENACT-2 trials respectively. During the ENACT-1 study, acute infusion reactions (any adverse event occurring within 120 minutes after the initiation of an infusion) occurred in $8 \%$ of patients in the placebo group and $11 \%$ of those who received natalizumab. The corresponding rates for the ENACT-2 study were $8 \%$ and $7 \%$. Hypersensitivity like reactions occurred in $2 \%$ of patients in the placebo group and $5 \%$ of those in the natalizumab group in ENACT-1 study. The corresponding rates were $1 \%$ and $3 \%$ in the ENACT-2 study. Acute infusion reactions included headache, urticaria and erythema. Although the incidence of acute infusion and hypersensitivity-like reactions was low, these events occurred more frequently among patients with persistent, but not transient antibodies against natalizumab. Concomitant use of immunosuppressive and corticosteroid therapy appeared to be moderately protective against antibody formation in both trials. There were no reported cases of lymphoma or tuberculosis during the studies.

\section{Progressive multifocal leukoencephalopathy (PML)}

PML is an opportunistic infection of the central nervous system caused by Jacob-Creutzfeldt (JC) polyoma virus which results in multiple foci of demyelination in the white matter and cerebral cortex, leading to severe and potentially fatal neurological dysfunction. ${ }^{26}$ Clinical symptoms include weakness or paralysis, vision loss, impaired speech, cognitive impairment and depending on the area of involvement a plethora of neurological symptoms and signs can manifest. The virus attacks the oligodendrocytes in the central nervous system which produce myelin and hence it progresses rapidly often leading to death. It is diagnosed by testing for JC virus DNA in the cerebrospinal fluid or in a brain biopsy specimen.

The classic histopathological hallmarks of PML include enlarged oligodendroglial nuclei at the border of areas of demyelination, giant, bizarre astrocytes and lipid laden macrophages that scavenge myelin debris. In situ hybridization or immuno-histochemical staining permits identification of the virus. Characteristic evidence of damage caused by the virus in the brain can also be detected by magnetic resonance imaging. PML almost invariably occurs in the setting of profoundly impaired cell mediated immunity in patients with AIDS, leukemia or in organ transplant recipients. There is no known cure or definitive therapy available for PML. AIDS patients treated with highly active anti retroviral therapy (HAART) after being diagnosed with PML tend to have a slightly longer survival time than in patients who were already on HAART and then developed PML. ${ }^{27}$ Other antiviral agents that have been studied as possible treatment options include cidofovir and interleukin-2 but the research is still preliminary.

The occurrence of PML in patients with Multiple Sclerosis and Crohn's disease treated with natalizumab was totally unexpected. Three patients out of 3000 who had participated in clinical trials of natalizumab for treatment of multiple sclerosis or Crohn's disease developed PML. ${ }^{28}$ The first case was a 23 -year-old male with relapsing-remitting multiple sclerosis diagnosed in 1997, on treatment with interferon beta since 1998 was enrolled in a double blind trial of natalizumab and interferon beta in 2002. The patient developed inappropriate behavior in November 2004 and eventually progressed to left hemiparesis, dysarthria and cognitive impairment. After receiving 28 infusions of natalizumab the last dose in December 2004 the patient stopped the study drug. He was not immune compromised and had no risk factors for HIV. Analysis of cerebrospinal fluid resulted in JC virus DNA detection and diagnosis of PML was confirmed by brain biopsy. He was treated with intravenous cidofovir with further worsening of neurological function following which intravenous cytarabine was initiated resulting in neurological improvement in 2 weeks. By the end of May 2005 he had improved motor and cognitive function but he still had disabling ataxia, cognitive impairment, mild neglect and left hemiparesis at the time of his most recent follow-up. ${ }^{29}$

A 46-year-old woman with relapsing-remitting multiple sclerosis died from PML after receiving 37 doses of natalizumab as part of a clinical trial in combination with interferon 
beta in 2005. She developed sensory motor deficits and cognitive impairment that progressed rapidly and was treated with pulsed solumedrol assuming that she had multiple sclerosis exacerbation. After extensive investigations the treating neurologist suspected PML and a CSF analysis for JC virus PCR was positive. This patient did not receive any definitive treatment for PML. ${ }^{30}$ A sixty year old patient with long-standing ileal Crohn's disease treated with natalizumab in March 2002 as part of the ENACT-1 trial presented to the emergency room in July 2003 with severe confusion and disorientation. He had received 3 monthly infusions of $300 \mathrm{mg}$ of natalizumab and then resumed in February 2003 for a relapse of Crohn's disease. He had been treated with multiple therapies including azathioprine (75 to $150 \mathrm{mg}$ ) which was discontinued 8 months before this presentation due to refractory anemia. This patient had no focal neurological signs at presentation except for being mentally slow. His brain MRI revealed non enhancing lesions in the right frontal lobe. Because of progressive deterioration in his condition a partial resection of right frontal lobe was performed and a diagnosis of astrocytoma was entertained based on pathological findings. The patient's condition worsened post operatively and treatment with corticosteroids was initiated. He continued to deteriorate rapidly and died 3 months later in December 2003. In March 2005 after the occurrence of PML in two patients with multiple sclerosis who had received natalizumab, this patient's course was re-examined. Examination of the formalin fixed tissue from the resected brain lesions confirmed the diagnosis of PML. ${ }^{31}$ A clear temporal relationship was identified between the monthly natalizumab infusions and the occurrence of JC virus replication. Although he had been treated with corticosteroids, infliximab and azathioprine, JC virus DNA appeared in the serum only after the re-introduction of natalizumab as monotherapy. ${ }^{31}$ The three cases confirm that PML is a rapidly progressive and potentially fatal disease associated with natalizumab usage. Similarities among the patients include the clinical presentation and the speed in progression of clinical symptoms and signs. All three patients were concomitantly receiving other immunosuppressive agents preceding the diagnosis of PML. A retrospective study conducted by Yousry et al of more than 3000 patients who received natalizumab for multiple sclerosis, Crohn's disease and rheumatoid arthritis found no new case of PML and suggested a risk of developing the disease is about one per thousand patients ( 0.2 to 2.8 per thousand) treated with natalizumab for a mean duration of 18 months. ${ }^{32}$

The exact pathogenesis for the development of PML in the setting of natalizumab therapy is still unclear. One hypothesis defended by some authors that PML occurs as a result of viral re-activation from within the brain and do not support the suggestion that JC virus is activated from its latent stage in the kidneys, to enter the blood and then seed the central nervous system which is the basis of hypothesis by Verbeeck and colleagues. ${ }^{33}$

Identification of reliable predictors for PML risk remains a high currently unmet need to improve the safety of natalizumab. There is no clear evidence that screening for JC virus in urine and plasma could improve the safety. ${ }^{34}$ Early detection of the disease and prompt removal of the drug should remain the goal of treating clinicians. The most reliable biomarker of PML short of brain biopsy is detection of JC virus DNA in the cerebrospinal fluid of infected patients. While this has a high specificity, sensitivity is modest, being undetectable in $10 \%$ to $40 \%$ of patients with active disease, with no evidence that it could be used as a predictive marker. ${ }^{35,36}$ The only other sensitive test is a brain MRI which virtually detects all clinical lesions. However PML is an aggressive disease and detection in the asymptomatic stage has rarely if ever been reported presumably because it becomes clinically manifest rapidly, often leading to death within a few months. ${ }^{37}$

Since reversal of immune system is key to the arrest of PML, early withdrawal of the drug and possible acceleration of drug elimination through plasma exchange ${ }^{38}$ may minimize harm. Patients must understand and accept the rare risk of disability or death from PML, as opposed to the burden of Crohn's disease when the decision to use natalizumab is entertained. The benefit is more likely to outweigh the risk among a population with Crohn's disease who have failed immunomodulator therapy and biological therapies who are either poor candidates or have refused surgery.

\section{Drug administration issues}

Natalizumab is only available through a special restricted distribution program called the "TOUCH" prescribing program. (Tysabri Outreach: Unified Commitment to Health). ${ }^{39}$ This was developed by the manufacturer, Elan, because of the risk of Progressive Multifocal leukoencephalopathy (PML). This program restricts the use of natalizumab to patients with refractory disease and those who have failed treatment or developed intolerance to immune modulators and or tumor necrosis factor inhibitors.

The primary goal of the "TOUCH" program is to minimize the risk of developing PML. Prescribers and their patients who are to receive this medication must be enrolled in the program. Pharmacies who supply and infusion sites 
that administer the medication must also be authorized by the program. The program offers more interaction for the patients with their treating doctors and nurses. Before each infusion four questions (Table 2) are read aloud and answered verbally by the recipient. These questions assist in identifying patients at higher risk of developing PML while on treatment with natalizumab through inquires about new neurological symptoms, co-existing illness (HIV, AIDS, leukemia, lymphoma, organ transplant) and the use of other immune suppressive medications. If any potential risk of the infusion is identified the site staff must contact the patients healthcare professional prior to proceeding with the infusion. ${ }^{39}$ Natalizumab is usually administered as an intravenous infusion of $300 \mathrm{mg}$ over a period of 1 hour, every 4 weeks in an authorized infusion center. It should not be administered as an intravenous push or bolus. Patients are observed during the infusion and for 1 hour after the infusion is complete due to the risk of acute infusion reactions. It should be discontinued in patients with Crohn's disease if therapeutic benefit has not been achieved by 12 weeks of induction therapy and also in patients who are unable to discontinue concomitant steroid use within 6 months of starting therapy with natalizumab.

\section{Cost}

Currently the approximate cost of natalizumab is US\$2,184 per vial for an annual cost of approximately US $\$ 28,400.40$

\section{Conclusion}

Crohn's disease is a chronic potentially disabling disease of the gastrointestinal system that is neither medically nor surgically curable. The treatment goals primarily revolve around induction and maintenance of remission, improvement of quality of life and minimizing long-term complications.

\section{Table 2 "TOUCH” program questionnaire}

I. Over the past month, have you had any new or worsening medical problems (such as a new or sudden change in your thinking, eyesight, balance, strength, or other problems) that have persisted over several days?

2. Do you have a medical condition that can weaken your immune system, such as HIV infection or AIDS, leukemia, or lymphoma, or an organ transplant, that may suggest that your body is not able to fight infections well?

3. In the past month, have you taken medications to treat cancer or MS or any other medicines that weaken your immune system?

4. In the past month, other than for the treatment of a recent relapse, have you taken any of the following medicines: Solu Medrol, methyl prednisone, Decadron, dexamethasone, Depo-Medrol, prednisone or other steroid medicines?
Newer biologic therapies have been shown to be highly effective and are likely to be used earlier in the disease course to improve prognosis.

Natalizumab is a novel agent that selectively inhibits leukocyte trafficking. It is approved for the treatment of moderate to severe non-stricturing, non-penetrating Crohn's disease. Its efficacy is supported by rigorous clinical trials. Its use is currently limited to patients who have not tolerated or failed treatment with other biological agents (antibodies to tumor necrosis factor). Its effectiveness in anti-TNF naïve patients is not known but there is little enthusiasm for study in this population since anti-TNF agents are effective and well tolerated. Natalizumab should not be used concomitantly with other immunological agents due to the potential but rare risk of developing PML. It is currently only available through a special restricted program called "TOUCH" for patients with Crohn's disease. Due to the lack of reliable markers for predicting the risk of PML, future research should identify patients who are most likely to benefit from natalizumab therapy, while minimizing the possibility of rare but serious adverse events and also in developing reliable markers for predicting these events.

The development of natalizumab has lead to preliminary testing of a humanized anti-alpha4 beta 7 integrin antibody (vedolizumab) which inhibits gut specific MADCAM 1 mediated leukocyte adhesion. This agent is currently being developed only for the treatment of Crohn's disease and ulcerative colitis. ${ }^{41}$ If effective and safe these gut-specific therapies will be tested as primary options and would expand the available treatment options for Crohn's disease.

\section{Disclosures}

The authors declare no conflicts of interest.

\section{References}

1. Lichtenstein G, Hanauer S, Sandborn W. Management of Crohn's disease in adults, Am J Gastroenterol. 2009:doi:10.1038/ajg 2008.168.

2. Feagan BG, Vreeland MG, Larson LR, et al. Annual cost of care for Crohn's disease: a payor perspective. Am J Gastroenterol. 2000;95: 1955-1960.

3. Bodger K. Cost of illness of Crohn's disease. Pharmacoeconomics. 2002;20:639-652.

4. Baumgart DC, Sandborn WJ. Inflammatory bowel disease:clinical aspects and establishing and evolving therapies. Lancet. 2007;12: 1641-1657.

5. Sastangi J, Silverberg MS, Vermeire S, Colombel JF. The Montreal Classification of inflammatory bowel disease:controversies, consensus and implications. Gut. 2006;55:749-753.

6. Sandborn WJ, Fazio VW, Feagan BG, et al. AGA technical review on perianal Crohn's disease. Gastroenterol. 2003;125:1508-1530.

7. Itzkowitz SH, Yio X. Inflammation and cancer IV. Colorectal cancer in inflammatory bowel disease:the role of inflammation. Am J Physiol Gastrointest Liver Physiol. 2004;287:G7-G17. 
8. Gillen CD, Walmsley RS, Prior P, et al. Ulcerative colitis and Crohn's disease: a comparison of the colorectal cancer risk in extensive colitis. Gut. 1994;35:1590-1592.

9. Loly C, Belaiche J, Louis E. Predictors of severe Crohn's disease. Scand J Gastroenterol. 2008;43:948-954.

10. Sutherland L, Singleton J, Sessions J, et al. Double blind, placebo controlled trial of metronidazole in Crohn's disease. Gut. 1991;32: 1071-1075.

11. Hanauer SB. Top-down vs step-up approaches in chronic inflammatory bowel disease:presumed innocent or presumed guilty. Nat Clin Pract Gastroent Hepatol. 2005;2:493.

12. Lichtenstein GR, Yan S, Bala M, et al. Infliximab maintenance treatment reduces hospitalizations, surgeries and procedures in fistulizing Crohn's disease. Gastroenterol. 2005;128:862-869

13. Gisbert JP, Panes J. loss of response and requirement of infliximab dose intensification in Crohn's disease: A review. Am J Gastroenterol. 2009;104:760-767.

14. Schnitzler F, Fidder H, Ferrante M, et al. Long term outcome of treatment with infliximab in 614 patients with Crohn's disease:results from a single centre cohort. Gut. 2009;58:492-500.

15. Sandborn WJ, Rutgeerts P, Enns R, et al. Adalimumab induction therapy for Crohn's disease previously treated with infliximab. Ann Int Med. 2007;146:829-838.

16. Lobb RR, Helmer ME. The pathopysiologic role of alpha 4 integrins in vivo. J Clin Invest. 1994;1722-1728.

17. Rutgeerts P, Ververmeire S, Van Assche G. Biological therapies for inflammatory bowel diseases. Gastroenterol. 2009;136:1182-1197.

18. Miller DH, Khan QA, Sheremata WA, et al. A controlled trial of natalizumab for relapsing multiple sclerosis. $N$ Engl J Med. 2003;53: 466-472.

19. Best WR, Becktl JM, Singleton JW, Kern F. Development of a Crohn's disease activity index. National Cooperative Crohn's disease study. Gastroenterol. 1976;70:439-444.

20. Targan SR, Feagen BG, Fedorak RN, et al. Natalizumab for the treatment of active Crohn's disease: Results of the Encore trial. Gastroenterol. 2007;132:1672-1683.

21. Sandborn WJ, Colombel JF, Enns R, et al. Natalizumab induction and maintenance therapy for Crohn's disease. $N$ Engl J Med. 2005;353: 1912-1925

22. MacDonald JK, McDonald JWD. Natalizumab for induction of remission in Crohn's disease (review). Cochrane Database Syst Rev Rev. 2007:CD006097.

23. Hymas JS, Wilson DC, Thomas A. Natalizumab therapy for moderate to severe Crohn's disease in adolescents. J Pediatr Gastroenterol Nutr. 2007;44:185-191.

24. Lewis JD. C-reactive protein: anti-placebo or predictor of response. Gastroenterol. 2005;129:1114-1116.

25. Sands BE, Kozarek R, Spainhour J, et al. Safety and tolerability of concurrent natalizumab treatment for patients with Crohn's disease not in remission while receiving infliximab. Inflamm Bowel Dis. 2007;13:2-11.
26. Brooks BR, Walker DL. Progressive multifocal leukoencephalopathy. Neurol Clin. 1984;2:299-313.

27. Berger JR, Pall L, Lanska D, Whiteman M. Progressive multifocal leukoencephalopathy in patients with HIV infection. J Neurovirol. 1998;4:59-68.

28. Berger JR, Koralnik I. Progressive multifocal leukoencephalopathy and natalizumab - unforeseen consequences. $N$ Engl J Med. 353;4: 414-416.

29. Kleinschmidt-Demasters BK, Tyler KL. Progressive multifocal luekoencephalopathy complicating treatment with natalizumab and interferon beta-1a for multiple sclerosis. $N$ Engl J Med. 2005;353: 369-374.

30. Annette LG, Scott WA, Ari JG, Andrew WB, Daniel P. Progressive multifocal leukoencephalopathy in a patient treated with natalizumab. N Eng J Med. 2005;353:375-381.

31. Van Assche G, Van Ranst M, Sciot R, et al. Progressive multifocal leukoencephalopathy after natalizumab therapy for Crohn's disease. N Engl J Med. 2005;353:362-368.

32. Yousry TA, Major EO, Ryschkewitsch C, et al. Evaluation of patients treated with natalizumab for progressive multifocal leukoencephalopathy. N Engl J Med. 2006;354:924-933.

33. Verbeeck J, Van Assche G, Ryding J, et al. Viral loads in patients with Crohn's disease treated with immunosuppression:can we screen for elevated risk of PML. Gut. 2008;57:1393-1397.

34. Clifford DB. Natlaizumab and PML: A risky business? Gut. 2008;57: 1347-1349.

35. McGuire D, Barhite S, Hollander H, et al. JC virus DNA in cerebrospinal fluid of human immunodeficiency virus infected patients. Predictive value for progressive multifocal leukoencephalopathy. Ann Neurol. 1995;37:395-399.

36. Marzocchetti A, Di Giambenedetto S, Cingolani A, et al. Reduced rate of diagnostic positive detection of JC virus DNA in cerebrospinal fluid in cases of suspected progressive multifocal leukoencephalopathy in the era of potent anti retroviral therapy. J Clin Microbiol. 2005;43: 4175-4177.

37. Hall CD, Dafni U, Simpson D, et al. Failure of cytarabine in progressive multifocal leukoencephalopathy associated with human immunodeficiency virus infection. $N$ Engl J Med. 1998;338:1345-1351.

38. Khatri B, Fox R, Koo A, et al. The effect of plasma exchange in accelerating clearance of natalizumab in patients with multiple sclerosis. Results of the PLEX study. [Poster] 23rd congress of the European Committee for Treatment and Research in Multiple Sclerosis (ECTRIMS). Oct 11-14, 2007; Prague.

39. The Tysabri TOUCH (package insert) prescribing program. Biogen Idec and Elan pharmaceuticals. www.tysabri.com.

40. What is the cost of Tysabri infusion? www.steadyhealth.com. Posted 05/31/2008

41. Feagan BG, Greenberg GR, Wild G, et al. Treatment of active Crohn's disease with MLN0002, a humanized antibody to the alpha4 beta7 integrin. Clin Gastoenterol Hepatol. 2008;6:1370-1371.
Therapeutics and Clinical Risk Management

\section{Publish your work in this journal}

Therapeutics and Clinical Risk Management is an international, peerreviewed journal of clinical therapeutics and risk management, focusing on concise rapid reporting of clinical studies in all therapeutic areas, outcomes, safety, and programs for the effective, safe, and sustained use of medicines. This journal is indexed on PubMed Central, CAS,
Dovepress

EMBase, Scopus and the Elsevier Bibliographic databases. The manuscript management system is completely online and includes a very quick and fair peer-review system, which is all easy to use. Visit http://www.dovepress.com/testimonials.php to read real quotes from published authors. 